\title{
Digital leben: Agrar 4.0 - Einbindung digitaler Elemente in Unterrichtsmaterialien über Digitalisierungstendenzen im Agrarbereich
}

\author{
andrea.kircher@sbg.ac.at, Fachbereich Geographie und Geologie, Universität Salzburg \\ "*martin.anzengruber@sbg.ac.at, Fachbereich Geographie und Geologie, Universität Salzburg; HTL Ried im Innkreis
}

eingereicht am: 19.12.2018, akzeptiert am: 28.04.2019

Der Agrarbereich, als wichtiger, wenn auch kleiner Teil der österreichischen Wirtschaft, befindet sich im Wandel. Die fortschreitende Digitalisierung bringt wesentliche Veränderungen und Chancen hinsichtlich nachhaltiger Bewirtschaftung. Ausgehend von einer kleinen Schulbuchanalyse wurden zum Thema „Agrar 4.0“ eine eigene Doppelseite sowie ein Praxisblatt mit Aufgaben konzipiert. Dabei kommen auch digitale Inhalte zum Einsatz.

Keywords: Agrar 4.0, Digitalisierung, Nachhaltigkeit, Bildungswesen

Digital living: Agriculture 4.0 - Inclusion of digital elements into teaching materials about digitalization trends in the agricultural sector

Although the agricultural sector is only a small part of Austria's economy, it is important and it is undergoing transition. Progressive digitalization brings along significant changes and chances in regard to sustainable cultivation. Based on a little analysis of schoolbooks, a double page and a practice sheet with tasks were developed on the subject of „Agriculture 4.0“. It also makes use of digital content.

Keywords: Agriculture 4.0, digitalization, sustainability, education

\section{$1 \quad$ Einleitung}

Unter dem Schlagwort „Agrar 4.0“ möchten wir uns mit einer aktuellen Entwicklung im Agrarbereich auseinandersetzen. Schon seit geraumer Zeit hält die Digitalisierung Einzug in die Landwirtschaft und regt dabei neue Formen der Bewirtschaftung an. Diese prägen maßgeblich das Bild der Landwirtschaft und sollten somit in der Vermittlung gegenüber Schülerinnen und Schülern nicht fehlen. Dabei soll ganz klar auf Nachhaltigkeit Bezug genommen werden, welche vielfach bereits in den Lehrplänen für „Geographie und Wirtschaftskunde" verankert ist (vgl. BMB 2016).

Als exemplarischer Lebensbereich, in dem die Digitalisierung aktuell massive Veränderungen bewirkt, hat der Agrarbereich eine gewisse Brisanz. Deshalb soll hier in Form von Schulbuchseiten eine Aufbereitung dieser Thematik erfolgen. Gedacht sind die Schulbuchseiten für Schüler/innen der Sekundar- stufe II, weshalb die Arbeitsaufgaben gemäß dem österreichischen Kompetenzmodell (BMUKK 2012) mit Operatoren formuliert wurden.

\subsection{Angestrebte Ziele und Schulbezüge}

Im Vordergrund der Thematik „Agrar 4.0“ sollen vor allem Aspekte der Nachhaltigkeit stehen, wobei hier auf das Konzept der Bildung für nachhaltige Entwicklung (Lebensministerium 2002, BMUKK et al. 2008) verwiesen sei, welches in Österreich am 30. April 2002 beschlossen wurde. Ausgehend von UNESCO-Leitlinien wurden Handlungsfelder wie Umweltschutz und ländliche Entwicklung definiert. Österreich möchte sowohl bei Lernenden als auch bei Lehrenden bewusstseinsbildend wirken und verankert die Strategie der BNE im Bildungssystem. Die konzipierten Schulbuchseiten greifen inhaltlich dabei diverse Leitziele auf - u. a. Leitziel 10, welches für die 
Stärkung nachhaltiger Produkte und Dienstleistungen steht. In diesem Zusammenhang sei auch auf das Unterrichtsprinzip „Umweltbildung“ (BMBWF 2014) verwiesen. Bei der Weiterentwicklung der österreichischen Lehrpläne (vgl. Koller o. J.) setzten sich Hinsch et al. (2014) neben der Entwicklung von Kompetenzen gemäß BMUKK (2012) auch mit Basiskonzepten für Geographie und Wirtschaftskunde auseinander. So bietet jenes der „Nachhaltigkeit und Lebensqualität" eine Herangehensweise für den Unterricht bei der der Begriff im Sinne einer „ökologischen Modernisierung" (Hinsch et al. 2014: 53) aufgefasst wird und die Mensch-Umwelt-Beziehungen einfließen.

Neben dem Aspekt der Nachhaltigkeit soll auch ein Beitrag zur Digitalisierungsstrategie im Bildungsbereich geleistet werden - in Österreich beispielsweise in Form der „Digitalen Grundbildung“ (BMBWF 2018b). Bereits in der Unterstufe sollen Schüler/innen mit Digitalisierung vertraut werden - z. B. auch integriert in den Geographie- und WirtschaftskundeUnterricht. Anknüpfungspunkte bieten dann etwa der Kompetenzbereich „Gesellschaftliche Aspekte von Medienwandel und Digitalisierung". Kompetenzmodelle wie digi.komp (BMBWF 2018a) beinhalten das kritische Hinterfragen des Nutzens von digitalen Medien für Leben und Gesellschaft, welches in der Sekundarstufe II dementsprechend aufgegriffen und vertieft werden kann.

Ein grundlegendes Ziel bildet zudem die Stärkung der Bedeutung und des Wissens um die Zukunftsfähigkeit des Agrarbereichs. Schüler/innen sollen durch die Auseinandersetzung eigene, tiefere Einsichten gewinnen und sich der Relevanz dessen bewusst werden. Im Zuge der Digitalisierung der Landwirtschaft eröffnen sich veränderte und neue Berufe, sodass Schüler/ innen potentielle Bildungswege für sich erschließen können. Durch das Aufzeigen von Chancen, aber auch Risiken der aktuellen Entwicklungen, soll eine differenzierte Sichtweise geschaffen werden. Nicht zuletzt sollen Schüler/innen zu kritischen, mündigen Konsumentinnen und Konsumenten werden. Die Aufgaben des Agrarbereichs umfassen nämlich weitaus mehr als die Produktion von Lebensmitteln. Sie haben weitreichende Folgen, auch für andere Wirtschaftsbereiche und werden oft nicht über den Produkterlös abgegolten - vor allem in alpinen Gebieten. In diesem Sinne dürfen die Mehrleistungen eines funktionierenden Agrarsektors nicht außer Acht gelassen werden, denn neben Umweltschutz und Erhaltung der Biodiversität steht auch die Minimierung von Gefahrenpotentialen - man denke beispielsweise an Schutzwälder und Erosionsschutz. Um ökologische, ökonomische, technisch-technologische und soziale Aspekte zusammenzubringen, können interdisziplinäre Ansätze hilfreich sein, die durch Arten des fächerübergreifenden
Unterrichts realisiert werden oder aber auch zur Gänze vom Fach Geographie und Wirtschaftskunde selbst getragen werden können.

\subsection{Gestaltung von Schulbuchseiten}

Unter den zahlreichen Unterrichtsmedien findet das Schulbuch eine breite Anwendung im Unterricht. Einerseits liefert es fachspezifische Informationen sowie Materialien für den Unterricht. Andererseits bietet ein Schulbuch auch Anregungen für weiterführende Betrachtungen. Christian Sitte (2001: $448 \mathrm{ff}$.) befasst sich u.a. mit den unterschiedlichen Funktionen von Schulbüchern.

Grundsätzlich liegt eine Strukturierungsfunktion vor, d.h. Inhalte des Lehrplans erfahren eine gewisse Einteilung - nicht umsonst spricht man auch vom „heimlichen Lehrplan“. Aber auch für Lehrer/innen bieten sich thematische Anhaltspunkte und im Sinne einer Steuerungsfunktion mögliche Unterrichtsverläufe (etwa durch Aufgabenstellungen). Das Angebot an diversen Materialien fällt unter die Repräsentationsfunktion, wobei vor allem auch der Einbezug aktueller methodischer und didaktischer Praktiken erfolgen sollte (wie die zunehmende Nutzung digitaler Inhalte). Eine ansprechende Gestaltung trägt zur Motivationsfunktion bei. Für eine bessere Lesbarkeit wurde der Text bei den hier vorliegenden Schulbuchseiten in Spalten abgefasst. Die Differenzierungsfunktion hängt maßgeblich von der Auswahl der Inhalte $\mathrm{ab}$, die auf die unterschiedlichen Interessen der Schüler/innen abzielen. Bei der Übungs- und Kontrollfunktion wird auf die Arbeitsaufträge im Schulbuch Bezug genommen.

Sitte (2001: 450-453) beschreibt weiters unterschiedliche Schulbuchtypen, wobei im vorliegenden Fall die Wahl auf eine Mischung aus Lehr- und Arbeitsbuch fiel - für Österreich nicht untypisch. Zu den Aufgaben liegt immer auch ein Erwartungshorizont vor, der in einem Lehrer/innenbegleitheft bereitgestellt werden könnte. Beim Entwurf der Arbeitsanweisungen wurde darauf geachtet, alle drei Kompetenzniveaus gemäß des Kompetenzmodells für Geographie und Wirtschaftskunde (BMUKK 2012) abzudecken und eine Dominanz rein reproduktiver Aufgaben zurückzudrängen. Auch eine methodische Vielfalt bei den Aufgaben ist erstrebenswert. Inhaltlich stehen an erster Stelle Aktualität sowie fachliche Korrektheit der dargelegten Themen, weshalb im konkreten Fall das Augenmerk auf den (technologischen) Entwicklungen im Agrarbereich lag, ohne dabei jedoch die Gesamtheit der österreichischen Landwirtschaft aus dem Blick zu verlieren. Daneben gilt es, eine Anpassung an die sprachlichen Fähigkeiten der Schüler/innen zu finden, sodass dennoch ein fachliches Niveau einge- 
halten wird. Das schließt etwa die Verwendung von Fachbegriffen mit ein.

Bei einem Thema, welches die Digitalisierung sogar im Titel trägt, bietet sich die Einbindung digitaler Elemente quasi schon an. Durch Verweise in Form von Links und QR-Codes können auch auf Papier nicht abbildbare Inhalte, wie Videos, eingebunden werden. Unterschiedliche Apps sollen die Schüler/innen einladen, sich selbstständig damit zu befassen. Auch Sitte (2001: 467) stellt fest, dass ein Schulbuch niemals nur fertige Lernergebnisse beinhalten sollte, sondern vielmehr Anregungen für eine weiterführende Beschäftigung bieten muss.

Die beigegebenen Schulbuchseiten und das Praxisblatt sollen ein Angebot für Lehrer/innen darstellen. Entsprechend der recht umfangreichen Thematik rund um die Digitalisierung in der Landwirtschaft bestehen schlussendlich vielfältige Möglichkeiten für die konkrete inhaltliche und methodische Umsetzung im Unterricht.

\subsection{Die „Landwirtschaft“ in österreichischen Schulbüchern}

Obwohl Österreich längst kein Agrarland per Definition mehr ist, so besteht hierzulande doch eine große Relevanz der Landwirtschaft. Auch in der Außenwirkung spielen unsere Landschaft und entsprechend deren Bewirtschaftung eine Rolle.

Es folgt nun eine kleine Schulbuchanalyse, die ausschnittsweise zeigen soll, wie österreichische Schulbücher die Landwirtschaft thematisieren und welchen Raum welche Inhalte dabei einnehmen. Dazu wurden acht Exemplare (Germ et al. 2013, Heriszt et al. 2013, Hitz et al. 2012, Hitz et al. 2013, Malcik et al. 2013, Spreitzhofer \& Wagner 2014, Wohlschlägl 2006, Wohlschlägl 2008) unterschiedlicher Verlage und Schulstufen herangezogen.

Der Agrarbereich ist vergleichsweise unterrepräsentiert. Die Schulbücher zielen - so z. B. beim Wirtschaftsstandort Österreich - hauptsächlich auf Industrie und Tourismus ab. Meistens werden der Landwirtschaft zwei bis drei Seiten gewidmet, wenn überhaupt. Vereinzelt finden sich (mehr oberflächliche) Absätze und Randhinweise auch in anderen Kapiteln. Landwirtschaft wird immer im Zusammenhang mit Ressourcen (Verfügbarkeit, schonender Umgang, etc.) oder dem menschlichen Eingriff und den damit einhergehenden Veränderungen der landschaftsökologischen Zonen dargelegt. Mit technologischen Entwicklungen setzt man sich in den gesichteten Exemplaren nicht auseinander. Sie werden allenfalls angesprochen, ihr Einsatz und ihre Entwicklung jedoch nicht aufgezeigt. Von daher stellt diese Konzeption eine Neuheit dar. Herausgestochen hingegen ist
Meridiane 7 (Hitz et al. 2013), das nach fünf Seiten zur Landwirtschaft auch noch vier Seiten enthält, die sich explizit und ausführlich der biologischen Landwirtschaft widmen.

\section{Thema: Agrar 4.0}

In vielen Bereichen hat die Digitalisierung schon Einzug gehalten, sei es nun in der Industrie, in zahlreichen Wirtschaftszweigen oder in der Schule. Auch der Agrarbereich befindet sich in einer derartigen Entwicklung. Digitalisierung passiert dabei in zwei Ausprägungen: Zum einen werden Aufzeichnungen digital statt analog erfasst, zum anderen erfolgt eine Automation von Prozessen und Geschäftsmodellen, indem Informationen miteinander vernetzt werden (BMEL 2017). In gewisser Weise finden sich Parallelen zur Industrie 4.0 - daher auch die Bezeichnung Agrar 4.0 -, wobei jedoch wesentliche Aspekte nicht übertragbar sind. So lassen sich Tiere und Pflanzen auf keine Norm beziehen, wie es bei technischen Bauteilen der Fall ist. Ebenso stellen klimatische Bedingungen und das Wettergeschehen Einflüsse dar, die nicht in der Hand der Menschen liegen.

Als Informationsgrundlage sei hier auf eine Publikation des Bundesministeriums für Tourismus und Nachhaltigkeit (BMNT 2018a) verwiesen, die sich umfassend mit der Digitalisierung im Agrarbereich befasst. Wohin führt nun diese Digitalisierung? Wesentlicher Bestandteil in der zukünftigen landwirtschaftlichen Produktion werden Daten sein, die etwa via GPS und Sensoren an den diversen Landmaschinen gesammelt werden. Die Aufzeichnungen können mittels digitaler Werkzeuge wie FARMDOK (https://www. farmdok.com/) direkt am Feld vorgenommen werden. GIS-Anwendungen helfen, die verschiedenen Daten zusammenzuführen und durch Analysen neue Informationen zu generieren. So dienen Ertragsmessungen als Anhaltspunkt für die Ausbringung von Düngemitteln, aber auch Investitionsentscheidungen können bei bekannten Auslastungen fundierter getroffen werden.

Nachdem vor rund 100 Jahren der erste benzinbetriebene Traktor übers Feld rollte, steht heute ein breites Spektrum an Technik und Technologie zur Verfügung. Mit der Unterstützung durch Drohnen und Kameras können Daten gewonnen werden, aber auch etwa beim Pflanzenschutz finden sie konkret Anwendung. Weiters gibt es Melkroboter, welche die Kühe selbst aufsuchen können und die sich mit ausgeklügelter Sensorik an jedes einzelne Tier anpassen. Im Ackerbau navigieren Landmaschinen auf gespeicherten Routen von selbst - und das zentimetergenau. Durch ihre Vernetzung und Follow-Funktionen werden Erntevorgänge automatisiert. 


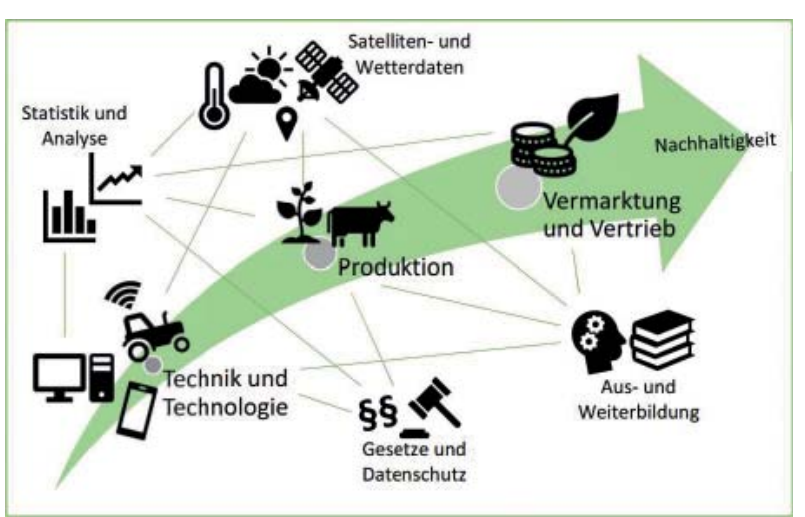

Abb. 1: Agrar 4.0

Trotz der Chancen, die durch die neuen Technologien entstehen, werden Stimmen laut, die auf die Risiken der Digitalisierung hinweisen. Big Data ist längst im Agrarbereich angekommen und damit auch die Frage der Datenhoheit. Derzeit sind lediglich personenbezogene Daten geschützt. Bei Maschinendaten sind die Besitzverhältnisse derzeit schlecht bis gar nicht geregelt, was das Betriebsgeheimnis infrage stellt. Kein Wunder also, wenn landwirtschaftliche Betriebe eine Überwachung befürchten. Es wird klare Regeln brauchen.

Man hat zudem erkannt, dass der Bereich der Ausund Weiterbildung enorm wichtig ist. Zukünftig werden sich die Berufsbilder im Agrarbereich wesentlich ändern. Es werden neue Ausbildungsmöglichkeiten und ganze Studiengänge geschaffen - nicht zuletzt, um eine Höherqualifizierung und somit eine Aufwertung des Berufs Landwirt/in zu erreichen.

Auch deshalb sollten bereits in der Schule der Agrarbereich und dessen Entwicklung aus einer moderneren Perspektive betrachtet werden, sodass sich das gesellschaftliche Bild der Landwirtschaft verändern kann. Das Bewusstsein um die menschlichen Lebensgrundlagen scheint vielfach in den Hintergrund getreten zu sein. Die ständige Verfügbarkeit von billigen Lebensmitteln sollte keineswegs eine Selbstverständlichkeit sein, sondern vielmehr eine angemessene Wertschätzung erhalten. Die Landwirtschaft steht vor vielen Herausforderungen, die teils durch die Digitalisierung gemeistert, aber teils auch erst durch sie verursacht werden. Eine wichtige Botschaft für die Schule besteht weiters in dem Wissen, warum Digitalisierung nicht immer nur breite Akzeptanz findet. Unter anderem geht es darum, die Sicherheit der gesammelten Daten zu bedenken. Denn wer die Datenhoheit hat, besitzt Macht und kann dementsprechend Einfluss ausüben.

Die Auseinandersetzung mit der Zukunft der Landwirtschaft zeigt auf, dass man beginnt, langfristig zu denken. Gefragt sind Konzepte mit nachhaltiger Bewirtschaftung. Die biologische Landwirtschaft in
Verflechtung mit Digitalisierung kann dabei ein Beispiel für nachhaltige Entwicklung darstellen. So eröffnen sich auch für Österreich und seine kleinstrukturierte Landwirtschaft Zukunftschancen. Im Bereich der konventionellen Landwirtschaft profitiert man von neuen Möglichkeiten hinsichtlich eines präziseren und reduzierten Betriebsmitteleinsatzes.

\section{Arbeitsaufträge}

Die Arbeitsaufträge auf den beigegebenen Schulbuchseiten und dem Praxisblatt sollen eine Mischung aus den drei Anforderungsbereichen des österreichischen Kompetenzmodells aus Geographie und Wirtschaftskunde (BMUKK 2012) darstellen und wurden dementsprechend mit den Operatoren (vgl. Sitte 2011) formuliert. Sie sollen jedenfalls über das Reproduzieren von Informationen hinausgehen. Die Schüler/ innen sollen angeregt werden, die dargebotenen Informationen um eigene Entdeckungen zu ergänzen und sich so in die Thematik zu vertiefen. Die Arbeitsaufträge sollen den Schülerinnen und Schülern zudem einen gewissen Freiraum für durchaus kreative Lösungen lassen. Es bestehen vielfältige Möglichkeiten zur Vertiefung, die Interessierte wahrnehmen können.

Die erste Aufgabe besteht darin, das Programm ÖPUL zu recherchieren und sich mit dessen Zielen auseinanderzusetzen. Dadurch sollen die Schüler/ innen einen Eindruck gewinnen, welche Bedeutung einer umweltschonenden Bewirtschaftung hierzulande beigemessen wird und wie agrarische Richtlinien in Programme münden. Insbesondere stellt das Ziel „Förderung der Innovation, der Zusammenarbeit und des Aufbaus der Wissensbasis in ländlichen Gebieten “ (BMNT 2018b) eine Verbindung zur Entwicklung rund um Agrar 4.0 her. Weiters soll offenbar werden, dass zwischen Landwirtschaft und Tourismus Zusammenhänge bestehen.

In der zweiten Aufgabe sollen mindestens vier Kriterien für biologische Landwirtschaft genannt werden, die nicht schwer zu finden sind. Ziel ist die Erkenntnis, dass sich die Biolandwirtschaft von der konventionellen Landwirtschaft in ihren Bewirtschaftungsweisen maßgeblich unterscheidet. Trotz teils sehr strenger Auflagen entscheiden sich Betriebe für Bio und das zeigt sich in der Bedeutung - auch für den heimischen Markt.

Die Schüler/innen sollen sich in der dritten Aufgabe, die direkt anschließt, mit der Bedeutung der Biolandwirtschaft auseinandersetzen und sich über ihr eigenes Konsumverhalten klarwerden. Ziel hierbei ist, die Schüler/innen zu einem bewussteren Blick zu animieren und sie für Meinungen zu wappnen, die nicht ihren eigenen entsprechen müssen. 
Die Randspalte enthält ergänzend einen Arbeitsauftrag zu Biogütesiegeln. Die Problematik besteht darin, dass am Markt bereits derartig viele Siegel kursieren und diese gewisse Erwartungen bei den Konsumentinnen und Konsumenten wecken. Die Aussagekraft von Biogütesiegeln wird dabei oftmals überschätzt. Bei genauerer Betrachtung zeigt sich nämlich, welche großen Unterschiede hinsichtlich der Kriterien bestehen. Die Schüler/innen sollen durch die Recherche ein Bewusstsein diesbezüglich erlangen und zu kritischen mündigen Konsumentinnen und Konsumenten werden.

Die folgenden Aufgaben widmen sich mehr den technischen Komponenten von Agrar 4.0. So soll nach Landtechnik mit Teilbreitenschaltung und einem digitalen Herdenmanagement gesucht werden. Damit bekommen die Schüler/innen einen Eindruck, was technisch möglich ist bzw. auch bereits eingesetzt wird.

Das Praxisblatt widmet sich ergänzenden sowie vertiefenden Aufgaben, welche teilweise online als App absolviert werden, die leicht über den QR-Code oder den angegebenen Link aufgerufen werden können. Es besteht hier auch die Möglichkeit am Computer, auf Tablets oder mit Smartphones zu arbeiten.

In der Aufgabe zur Smart Farm (A 1) soll aufgezeigt werden, wie ein Agrarbetrieb vernetzt sein kann. Dabei werden wesentliche Aspekte aus den vorangehenden Seiten aufgegriffen und sollen auch durch die grafische Darstellung gefestigt werden.

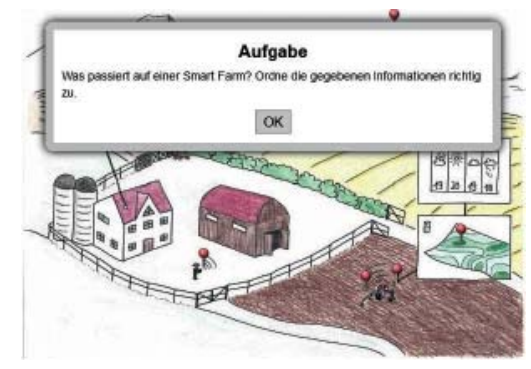

Abb. 2: Learningapp "Smart Farm“

Das Schätzspiel (A 2) soll das Gefühl für den Agrarbereich und seine Ausmaße vermitteln. Vor allem sollen auch anschauliche Zahlenbeispiele die Bedeutung des Biomarktes und der diesbezüglichen Stärke Österreichs begreiflich machen - immerhin rangiert Österreich in Bio-Statistiken häufig unter den Top 10.

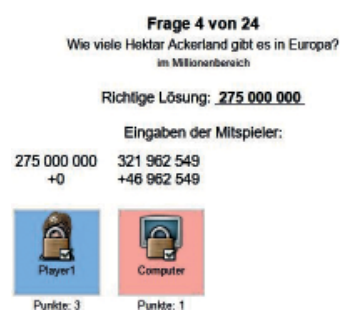

Abb. 3: Learningapp „Daumen mal Pi“

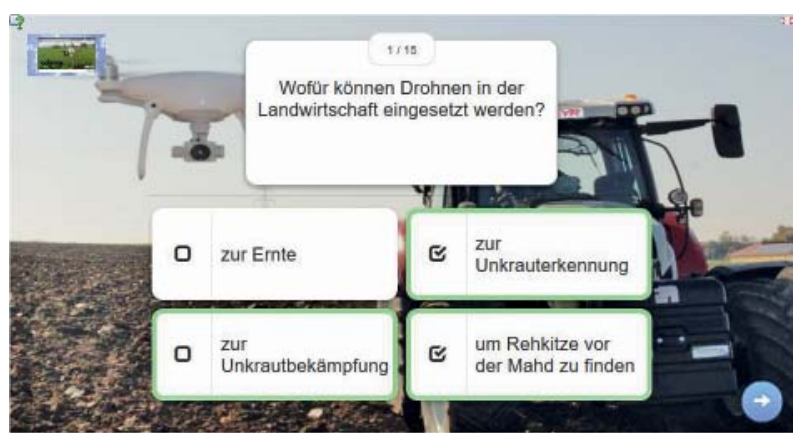

Abb. 4: Quiz zum Drohneneinsatz in der Landwirtschaft

Der Drohneneinsatz als technologischer Aspekt bietet vielfältige Möglichkeiten für den Agrarbereich. So geht es in A 3 darum, Einsatzbereiche für Drohnen in der Landwirtschaft in Erfahrung zu bringen. Das Quiz dient dann als lockere Wiederholung zum zuvor betrachteten Video und muss nicht zwingend absolviert werden. Wieder ist es durch den QR-Code oder den Link leicht aufrufbar und eignet sich auch für die Bearbeitung auf mobilen Endgeräten.

Das Prozessschaubild in A 4 (Abb. 1) setzt die einzelnen Aspekte und Teilbereiche von Agrar 4.0 miteinander in Beziehung. Durch das Formulieren einer eigenen Beschreibung sollen sich die Schüler/innen eingehender mit den Verbindungen auseinandersetzen und die davor gelernten Inhalte noch einmal strukturiert betrachten. Das Ergänzen der Praxisbeispiele soll helfen die einzelnen Bereiche und ihre Vernetzung und Wechselwirkungen untereinander zu illustrieren.

Die Schüler/innen sollen sich in A 5 konkret mit den Bedingungen für einen Bio-Betrieb auseinandersetzen und der Frage auf den Grund gehen, warum viele die nicht unbeschwerliche Umstellung auf sich nehmen. Zudem geht es um Überlegungen, wie die Digitalisierung hier von Vorteil sein könnte, indem die Schüler/innen beispielsweise zuvor gelernte Aspekte aufgreifen.

Die Aufgabe A 6 stellt einen möglichen Abschluss der Thematik rund um Agrar 4.0 dar und erfordert an sich keine Bearbeitung mit Output. Als eine Form der Nachbereitung kann die Zukunft der Landwirtschaft jedochim Plenumaufgegriffen und besprochen werden. So lassen sich auch eventuell noch offene Fragen klären.

$\mathrm{Da}$ „Agrar 4.0“ weit mehr umfasst, als auf ein paar Seiten abgebildet werden kann, möchten wir noch ein paar weitere Ideen darlegen. Die Involviertheit der Schüler/innen kann auch gut durch eigene Erfahrungen erreicht werden. Deshalb sollen sie sich beispielsweise auch Gedanken über ihr eigenes Konsumverhalten machen. Man kann aber auch der Frage nachgehen, wer in der Klasse einen landwirtschaftlichen Hintergrund besitzt und wie weit dieser zurückreicht, Stichwort Sektoraler Wandel (Eltern, Großeltern, ...). 
Schüler/innen könnten weiters Freude daran haben, selbst Bio-Verpackungen zu inspizieren und der Herkunft und Produktion der Produkte nachzugehen.

\section{$4 \quad$ Feedback und Adaptionen}

Die Schulbuchseiten wurden in einer Art Feldtestung Schülerinnen und Schülern des 3. und 4. Jahrgangs der HTL Ried ${ }^{1}$ vorgelegt. Sie durften sich in Eigenarbeit mit den Seiten beschäftigen und lieferten anschließend wertvolle erste Erfahrungsberichte.

Erfreulicherweise kommen gerade die digitalen Inhalte ausgesprochen gut an - vor allem die Einbindung eines Quiz und eines Schätzspiels. Zu bemängeln sei jedoch, dass die Funktionsweise der QR-Codes nicht immer gegeben ist - gerade mit älteren Smartphones können Schwierigkeiten auftreten. Aus diesem Grund sind auch gekürzte Links angegeben. Zudem sollte die Anzahl der QR-Codes ein gewisses Maß nicht übersteigen. Die Schüler/innen stellen weiters fest, dass sich hinter den Links zu viele zusätzliche Inhalte „verstecken " können. So etwa ein interaktives Info-Tool der Bio Austria, welches deshalb herausgenommen wurde. Auch die ursprüngliche Aufgabe A 3, in welcher von der Deutschen Landwirtschaftsgesellschaft DLG geforderte Rahmenbedingungen für eine digitalisierte Landwirtschaft bewertet werden sollten, wurde durch eine Aufgabe zum Prozessschaubild (Abb. 1) ersetzt.

Durch die Strukturierung der Seiten fühlten sich die Schüler/innen gut durch die Thematik begleitet. Für manche Abkürzungen und Fremdwörter (z. B. Biodiversität) würden Erklärungen in der Randspalte gewünscht werden. Als für die Sekundarstufe II konzipierte Unterlage und im Hinblick auf die Reifeprüfung können gewisse Begriffe aber durchaus zugemutet werden, die im Bedarfsfall recherchiert oder bei der Lehrkraft nachgefragt werden können.

Ein häufig eingebrachter Kritikpunkt hinsichtlich Inhalten liegt in der Behandlung der Biolandwirtschaft - genauer gesagt deren Vor- und Nachteilen, die nicht explizit ausgeführt werden oder in einem Arbeitsauftrag zur Beschäftigung münden. Die Schüler/innen geben auch zu bedenken, dass die Digitalisierung nur positiv behandelt würde, sprich Kritikpunkte an der Entwicklung Agrar 4.0 fehlen, was in gewisser Weise beabsichtigt war. Die Digitalisierung sollte unter dem Aspekt der Zukunftsfähigkeit und den positiven Einflüssen digitaler Neuerungen behandelt werden. Unter anderem führen die Schüler/innen diesbezüglich an, dass die in Österreich kleinstrukturierte Landwirtschaft nicht unbedingt einen Nutzen

Die HTL für Maschinenbau führt seit 2016 den schulautonomen Schwerpunkt „Agrar- und Umwelttechnik“. aus den Modernisierungen ziehen kann (Stichwort Investitionskosten).

Aufgrund der Erfahrungen im Unterricht wurden auch missverständliche Textstellen umformuliert und stellenweise die Inhalte und Überleitungen noch präzisiert.

\section{Literatur}

BMB (Bundesministerium für Bildung) (2016): Änderung der Verordnung über die Lehrpläne der allgemein bildenden höheren Schulen. Bekanntmachung der Lehrpläne für den Religionsunterricht an diesen Schulen. BGBl. II Nr. 219/2016. URL: https://www.ris.bka.gv.at/ Dokumente/BgblAuth/BGBLA_2016_II_219/BGBLA_2016_II_219.pdf (12.8.2018)

BMBF (Bundesministerium für Bildung und Frauen) (2012): Die kompetenzorientierte Reifeprüfung Biologie und Umweltkunde. Richtlinien und Beispiele für Themenpool und Prüfungsaufgaben. URL: https://bildung. bmbwf.gv.at/schulen/unterricht/ba/reifepruefung_ahs_ lfbio_21976.pdf?Gaanmk (12.8.2018)

BMBF (Bundesministerium für Bildung und Frauen) (2015): Verordnung der Bundesministerin für Bildung und Frauen, mit der eine Verordnung über die Lehrpläne der Höheren technischen und gewerblichen Lehranstalten 2015 erlassen wird sowie die Verordnung über die Lehrpläne für Höhere technische und gewerbliche Lehranstalten und die Verordnung über die Lehrpläne der Höheren technischen und gewerblichen Lehranstalten (Lehrplan 2011) geändert werden (Lehrplanpaket der Höheren technischen und gewerblichen Lehranstalten 2015); Bekanntmachung der Lehrpläne für den Religionsunterricht, BGBl. II Nr. 262/2015, Anlage 1. URL: https://www4.lernplattform.schule.at/gwk/mod/url/ view.php?id=13094 (25.3.2019)

BMBWF (Bundesministerium für Bildung, Wissenschaft und Forschung) (2014): Grundsatzerlass Umweltbildung für nachhaltige Entwicklung. URL: https://bildung. bmbwf.gv.at/schulen/unterricht/prinz/ub/umweltbildung.html. (25.3.2019)

BMBWF (Bundesministerium für Bildung, Wissenschaft und Forschung) (2018a): digi.komp: Digitale Grundbildung in allen Schulstufen. URL: https://bildung.bmbwf.gv.at/schulen/schule40/digikomp/digikomp.html (7.9.2018)

BMBWF (Bundesministerium für Bildung, Wissenschaft und Forschung) (2018b): Digitale Grundbildung. URL: https://bildung.bmbwf.gv.at/schulen/schule40/dgb/index.html (7.9.2018)

BMEL (Bundesministerium für Ernährung und Landwirtschaft) (2017): Digitalpolitik Landwirtschaft. Zukunftsprogramm: Chancen nutzen - Risiken minimieren. URL: https://www.bmel.de/SharedDocs/Downloads/ Broschueren/DigitalpolitikLandwirtschaft.pdf?__blob =publicationFile (12.08.2018) 
BMNT (Bundesministerium für Nachhaltigkeit und Tourismus) (2018a): Digitalisierung in der Landwirtschaft. Entwicklung, Herausforderungen und Nutzen der neuen Technologien für die Landwirtschaft. URL: https:// www.bmnt.gv.at/dam/jcr:f80a7032-3cb3-422b-8fa3e3199d2caf4d/Digitalisierung\%20in\%20der\%20Landwirtschafti.pdf (14.12.2018)

BMNT (Bundesministerium für Nachhaltigkeit und Tourismus) (2018b): ÖPUL 2015 - das Agrar-Umweltprogramm bis 2020. URL: https:/www.bmnt.gv.at/land/laendl_entwicklung/oepul/oepul2015.html (12.08.2018)

BMUKK (Bundesministerium für Unterricht, Kunst und Kultur)/Lebensministerium (Bundesministerium für Land- und Forstwirtschaft, Umwelt und Wasserwirtschaft)/BMWF (Bundesministerium für Wissenschaft und Forschung) (2008): Österreichische Strategie zur Bildung für nachhaltige Entwicklung. URL: https:// bildung.bmbwf.gv.at/schulen/unterricht/ba/bine_strategie_18299.pdf?61ed8p (05.03.2018)

BMUKK (Bundesministerium für Unterricht, Kunst und Kultur) (2012): Die kompetenzorientierte Reifeprüfung aus Geographie und Wirtschaftskunde. Richtlinien und Beispiele für Themenpool und Prüfungsaufgaben. URL: https://bildung.bmbwf.gv.at/schulen/unterricht/ba/reifepruefung_flf.html (25.3.2019)

Germ, A., F. Hochreiner, G. Mayrhofer \& F. B. Part (2013): Geospots 7/8. Geografie und Wirtschaftskunde für die AHS, 1. Aufl. Veritas-Verlag, o. O.

Heriszt, A., F. Hochreiner \& G. Mayrhofer (2013): Geospots HAK (auch IWK), HAS, HLW, HTL für alle Jahrgänge, 6. Aufl. Veritas-Verlag, o. O.

Hinsch, S., H. Pichler, T. Jekel, L. Keller, \& F. Baier (2014): Semestrierter Lehrplan AHS, Sekundarstufe II. Ergebnis der ministeriellen Arbeitsgruppe. In: GW-Unterricht 136, S. 51-61. URL: http://www.gw-unter-richt.at/ images/pdf/gwu_136_51_61_hinsch_pichler_jekel_keller_baier.pdf, (12.8.2018)

Hitz, H., A. Kowarz, I. Kucera \& W. Malcik (2012): Meridiane 5/6. Lehr- und Arbeitsbuch Geographie und
Wirtschaftskunde für die 5. und 6. Klasse an allgemein bildenden höheren Schulen, 1. Aufl. Ed. Hölzel, o. O.

Hitz, H., A. Kowarz, I. Kucera \& W. Malcik (2013): Meridiane 7. Lehr- und Arbeitsbuch Geographie und Wirtschaftskunde für die 7. Klasse an allgemein bildenden höheren Schulen, 2. Aufl. Ed. Hölzel, o. O.

Koller, A. (о. J.): Lehrpläne und Basiskonzepte. In: Online-Fachdidaktik PH-Linz. URL: https://www4. lernplattform.schule.at/gwk/course/view.php?id=389 (25.3.2019)

Lebensministerium (Bundesministerium für Land- und Forstwirtschaft, Umwelt und Wasserwirtschaft) (2002): Österreichs Zukunft Nachhaltig Gestalten - Die Österreichische Strategie zur Nachhaltigen Entwicklung. URL: https://www.nachhaltigkeit.at/assets/customer/Downloads/Strategie/strategie020709_de.pdf (05.03.2018)

Malcik, W.,W. Sitte \& C. Sitte (2013): Raum - Gesellschaft - Wirtschaft 7 neu, akt. Bearb. 2013, Ed. Hölzel, o. O.

Sitte Ch (2001): Das GW-Schulbuch. In: Sitte, W., Wohlschlägel,H.(Hrsg.), Beiträgezur Didaktikdes „Geographie und Wirtschaftskunde“-Unterrichts. Wien (= Materialien zur Didaktik der Geographie und Wirtschaftskunde, Bd. 16), 4. unveränderte Auflage 2006, S. 447-472 Online: https://www.univie.ac.at/geographie/fachdidaktik/Handbuch_MGW_16_2001/inhalt_Handbuch_Geographie_und_Wirtschaftskunde2001.htm

Sitte, C. (2011): Maturafragen neu (!?) - eine schrittweise Annäherung an eine kompetenzorientierte Form im Fach Geographie und Wirtschaftskunde. In: GW-Unterricht 123, S. 36-41. URL: http://www.gw-unterricht.at/images/pdf/gwu_123_024_041_sitte.pdf (25.3.2019)

Spreitzhofer, G. \& H. Wagner (2014): Wissen-KönnenHandeln. Geografie (Wirtschaftsgeografie) HAK Jg. 1/2, 1. Aufl. o. O.

Wohlschlägl, H. (Hrsg.) (2006): Durchblick 7. Geographie und Wirtschaftskunde für die 11. Schulstufe, 1. Aufl. Westermann Wien, o. O.

Wohlschlägl, H. (Hrsg.) (2008): Durchblick 5. Geographie und Wirtschaftskunde, 4. Aufl. Westermann Wien, o. O. 
Neben den physischen Faktoren wirkt der Mensch mit wirtschaftlichen und politischen Entscheidungen auf unterschiedlichen Ebenen auf den Agrarbereich ein. Soziale Einflüsse und Meinungen prägen das gesellschaftliche Bild der Landwirtschaft.
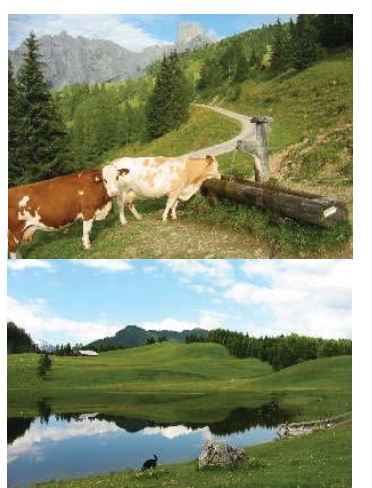

M 1 Almen sind ein Teil der landwirtschaftlichen Produktion in Österreich

Biolandwirtschaft Eine biologische Wirtschaftsweise steht für die Schonung natürlicher Ressourcen und unterliegt speziellen gesetzlichen Regelungen.

Statistiken bescheinigen Österreich den Status als "Bioland".

\section{Biogütesiegel}

> Informiere dich über Biogütesiegel. (z.B. www.bewusstkaufen.at)

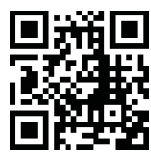

> Erkläre Gemeinsamkeiten und Unterschiede von mind. drei ausgewählten Siegeln. (Kriterien, wie streng?)

> Bewerte in zwei bis drei Sätzen die Aussagekraft von Biogütesiegeln.

\section{Agrar 4.0 - Digitalisierung in der Landwirtschaft}

Die Menschheit betreibt seit der neolithischen Revolution Landwirtschaft und trägt so zur Versorgung mit Lebensmitteln bei. Das Klima bestimmt dabei wesentlich, was angebaut werden kann, aber auch Boden und Relief sind relevante Einflussfaktoren sowie agrarpolitische Maßnahmen (z.B. Subventionen). Es findet nicht nur ein Strukturwandel hinsichtlich Betriebsgrößen statt. Vielmehr entstehen durch die zunehmende Digitalisierung ganz neue Berufsfelder. In diesem Zusammenhang spricht man auch von Agrar 4.0 - in Anlehnung an Industrie 4.0.

\section{Strukturen im Wandel}

Österreich weist in seinen landwirtschaftlichen Produktionsgebieten eine große Heterogenität auf. Neben benachteiligten Gebieten wie den Alpen (Klima und Topographie) gibt es auch besonders fruchtbare Regionen (z.B. Marchfeld und Alpenvorland). Typisch für Österreich sind zudem Bergbavernbetriebe, die widrige Lagen (z.B. Steilflächen, ungünstige Boden- und Klimaverhältnisse) und die Grenze zur Anökumene bewirtschaften. Sie erfüllen zudem wichtige Funktionen für den Tourismus.

Im Unterschied zu den anderen Wirtschaftssektoren wie der Industrie trägt der Agrarbereich nur wenig zur Wirtschaftsleistung bei. 2017 waren es laut Statistik Austria gerade einmal 1,2\% des österreichischen BIP. Hingegen verzeichnete man 1950 noch $19 \%$.

Für Österreichs vergleichsweise kleinbis mittelstrukturierte Land- und Forstwirtschaft ist die Einkommensvielfalt auf den Höfen typischerweise in $\mathrm{Ne}$ ben- und Haupterwerbsbetrieben organisiert. In den Hauptproduktionssparten der Urproduktion (Milch, Getreide, Fleisch, Gemüse, ...) sind die bäverlichen Familienbetriebe in einem internationalen Wettbewerb gefordert, bei offenen Märkten zu bestehen.
Daneben bieten sich Einkommenschancen mit Qualitätsprodukten, Direktvermarktung auf regionalen Märkten, Urlaub am Bavernhof, Diversifikation auf den Höfen und Dienstleistungsangeboten in einem erweiterten bäuerlichen Berufsbild sowie Nutzung der Einkommenskombination mit einem außerlandwirtschaftlichen Erwerb.

\section{Bedeutung und Nachhaltigkeit}

Die Landwirtschaft erfüllt außerordentlich wichtige Aufgaben für die Gesellschaft. Neben der Produktion von Nahrungsmitteln geht es auch um die Kulturlandschaftserhaltung und Biodiversität. Zudem ist die Land- und Forstwirtschaft in vor- und nachgelagerten Bereichen eng vernetzt mit anderen Wirtschaftszweigen (Tourismus), in der Be- und Verarbeitung von Rohstoffen, Bereitstellung von Betriebsmitteln, Maschinen, Geräten usw.

\footnotetext{
> Ermittle die Ziele des Programms ÖPUL (z.B.
} www.bmnt.gv.at).

Zunehmend erfolgt ein Wandel vom regionalen Versorger zum Unternehmer, der ökonomischen Zielen unterliegt. Diese und die Anpassung an globale Regeln erzeugen enormen Druck für den Agrarbereich. Sind Landwirte Opfer der Globalisierung? Nur steigende Effizienz ist keine Lösung.

\section{Chancen und Risiken}

Die Digitalisierung ist im Agrarbereich bereits sehr fortgeschritten. Damit können sich Landwirte zunehmend anderen Aufgaben widmen und erhalten durch den Zugriff auf ihre Daten Anhaltspunkte für zukünftige Entscheidungen. Die modernen Landmaschinen rufen sogar bei Bedarf selbst den Service, sodass ein Eingreifen kaum mehr notwendig sein wird, wie dies z.B. bei Melkroboteranlagen der Fall ist.

Kritisch hingegen sehen Landwirte die Datennutzung durch andere Unternehmen. Bisher unterliegen nur personenbezogene Daten dem Datenschutz. Bei Maschinendaten sind häufig die Eigentumsrechte nicht eindeutig geklärt. Befürchtet werden ein gläserner Betrieb und eine Überwachung von außen. 
Vielmehr gilt es, den Agrarsektor durch umfassende Aus- und Weiterbildung zU stärken und durch Qualifizierung sowie Spezialisierung zukunftsfit zu machen. Große Entwicklungschancen stecken in der Nachhaltigkeit - nicht zuletzt boomt auch die Biolandwirtschaft.

> Nenne mind. vier Kriterien für biologische Landwirtschaft. (z.B. www.bio-austria.at)

> Untersuche die Bedeutung der Biolandwirtschaft - z.B. hinsichtlich Konsum, Umsatz oder genutzter Fläche.

> Überlege, wie oft du selbst zu biologischen Lebensmitteln greifst und warum (nicht)?

\section{Digitalisierung und Innovationen}

Der Einsatz von Technologie hält auch im Agrarbereich Einzug und wird entscheidend zu dessen Zukunft beitragen. Auf Revolutionen wie den ersten Traktor vor rund 100 Jahren folgen GPS-Steuerungen, Melkroboter, Drohnen und vieles mehr. Neue Analysemethoden helfen, neves Wissen über die landwirtschaftlichen Prozesse $\mathrm{zu}$ gewinnen, die mittels never Technologien gewonnen werden - und das vielfach in Echtzeit. Mit Begriffen wie Precision Farming, Smart Farming und Farming 4.0 versucht man, die Phänomene zu fassen.

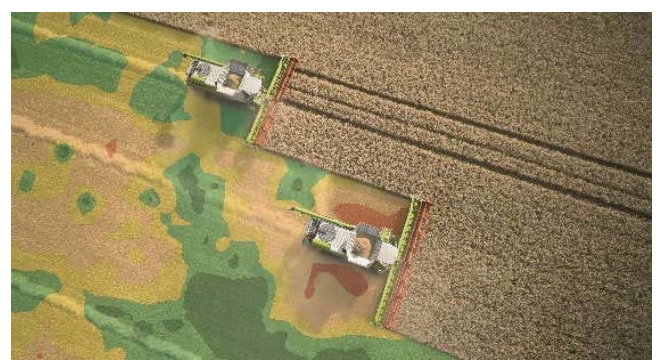

M 2 In der Präzisionslandwirtschaft werden Erträge auf landwirtschaftlichen Flächen erfasst. Mittels GIS und Applikationskarten kann dann der Einsatz von Düngemitteln gezielt erfolgen.

Die Analogie zur Industrie $\mathbf{4 . 0}$ ist in vielerlei Hinsicht gegeben, jedoch unterscheidet sich High-Tech auf dem Acker in einigen wesentlichen Punkten. Die vierten Revolutionen sind jeweils gekennzeichnet durch eine Vernetzung der Produktion mittels IKT Möglichkeiten, die durch das Internet entstehen.

Auch Mechanisierung, Motorisierung und Elektrifizierung spielen jeweils eine Rolle. Landwirtschaftliche Produktion passiert zum Unterschied jedoch nicht in wettergeschützten Werkshallen und bei Tieren und Pflanzen handelt es sich nicht um Normteile.

Wozu die Digitalisierung? Neben einer körperlichen Entlastung der Landwirte und einer Zeitersparnis profitiert auch die Umwelt von einer schonenderen Bewirtschaftung. Langfristig kann nächst einer Senkung der Kosten auch eine bessere Produktqualität erreicht werden.

Recherchiere im Web nach Landtechnik mit
Teilbreitenschaltungen (Section control).
Informiere dich über das digitale Herdenma-
nagement von SMARTBOW (z.B. www.smart-
bow.com).

\section{Zukunft der Landwirtschaft}

Österreich nimmt in der Entwicklung moderner Landtechnik eine wichtige Rolle ein. So stellte der Traktorenhersteller Lindner 2017 den autonom fahrenden Traktor Lintrac 110 vor. Die gesetzlichen Rahmenbedingungen müssen allerdings noch geschaffen werden, damit Innovationen wie die Funktion zum Folgen eines anderen Fahrzeugs in der Praxis zur Anwendung kommen können.

Quelle: ORF Tirol 25.10.2017, „Weltneuheit: Selbstfahrender Traktor"

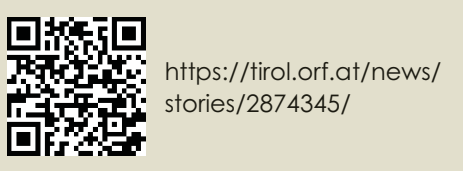

Ob sich eine Digitalisierung für einen land- und forstwirtschaftlichen Betrieb eignet, hängt derzeit an der Innovationsbereitschaft und Qualifikation der Betriebsleiter. Vor allem auch die Betriebsgröße und der Produktionsumfang bestimmen mit, ob diese neven Technologien ökonomisch sinnvoll eingesetzł werden können. Denkbar wären z.B. in der Berglandwirtschaft ein Einsatz von Melkrobotern oder Drohnen, um Herden in unwegsamem Gelände zu überwachen.

Bei allen technologischen Entwicklungen im Agrarbereich und auch in vorund nachgelagerten Wirtschaftsbereichen bedarf es qualifizierter Personen, die damit umzugehen wissen. In Zukunft werden ganze Studiengänge aufgebaut, in denen technische Schlüsselkompetenzen aus Informatik und Elektronik ebenso wie Logistik und Grundlagen des Ökolandbaus vermittelt werden.
Precision Farming

Präzisionslandwirtschaft bzw. teilflächenspezifische Bewirtschaftung

Durch Technologie (z.B. Infrarotsensoren und Kameras) können landwirtschaftliche Nutzflächen derart bewirtschaftet werden, dass Unterschiede hinsichtlich Bodenstruktur und Pflanzenbestand berücksichtigt werden.

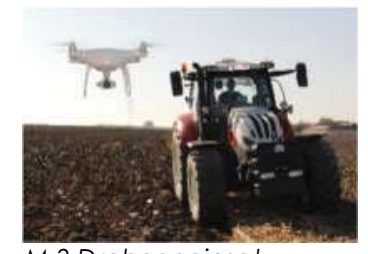

M 3 Drohneneinsatz

Smart Farming beinhaltet neben der Maschinensteuerung auch ein Bestands- und Datenmanagement, das von der Erfassung und Dokumentation von Arbeiten bis zur Verwendung der Daten in Planung und Verwaltung reicht.

Applikationskarten, Wetterinformationen und Echtzeitdaten machen kurzfristige Umplanungen möglich.

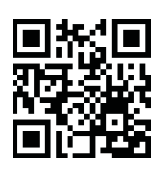

Smart Farming: High-Tech im Feld https://youtu.be/alvsMumLClA

Seit dem Wintersemester 2018 kann man an den $\mathrm{FHs}$ OÖ und Wiener Neustadt Agrartechnologie (und management) studieren. Weiters bietet die BOKU Wien zahlreiche Studiengänge im Umfeld des Agrarbereichs. 


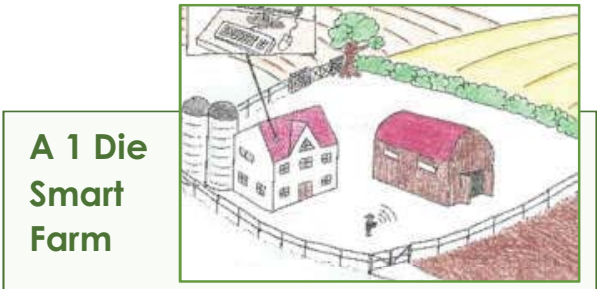

Ein moderner landwirtschaftlicher Betrieb kann Technologien einsetzen, um den heutigen Anforderungen zu begegnen. Landmaschinen arbeiten automatisiert und es kommt zu einer Vernetzung entlang der Wertschöpfungskette.

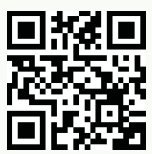

Digitale Landwirtschaft - die Smart Farm https://bit.ly/2EynrNQ

\section{A 3 Mit Drohnen gegen den Maiszünsler}

Drohnen können heutzutage bereits mehrere Kilogramm an Gewicht tragen. Mit befestigten Kameras können Überflüge Leben retten, aber auch Mittel zur Schädlingsbekämpfung können so punktgenau ausgebracht werden.

> Schau dir das Video dazu an und versuche dich am Quiz.

> Notiere konkrete Einsatzbereiche für Drohnen in der Landwirtschaft.

Video und Quiz:

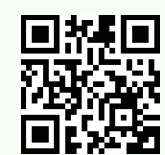

Drohnen in der Landwirtschaft

https://bit.ly/2QUyHcT

\section{A 2 Daumen mal Pi ...}

Ein Schätzspiel zur Landwirtschaft in Österreich

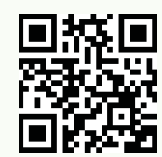

https://bit.ly/2BoOQNZ

\section{Weiterführende Links}

$>$ www.lko.at

$>$ www.lkdigital.at

$>$ www.bmnt.gv.at

$>$ www.statistik.at
Agrarische GIS-Anwendungen

In Österreich konnten bis 2015 über 250.000 Landwirte das AgrarGIS und somit professionelle GIS-Werkzeuge nutzen, um ihre Betriebe zu verwalten - mit Luftbildern, topographischen Karten sowie Fachkarten zu Klima, Boden, Schadensereignissen etc.

Aktuell stehen „Praxisplan Waldwirtschaft" und "Geokontakt" in Verbindung mit dem freien Programm QGIS zur Verfügung.

\section{A 4 Agrar 4.0 ...}

... umfasst neben technischen Innovationen und der Vernetzung von Maschinen und Informationen auch die Ausbildung sowie rechtliche Rahmenbedingungen und Fragen zur Nachhaltigkeit.

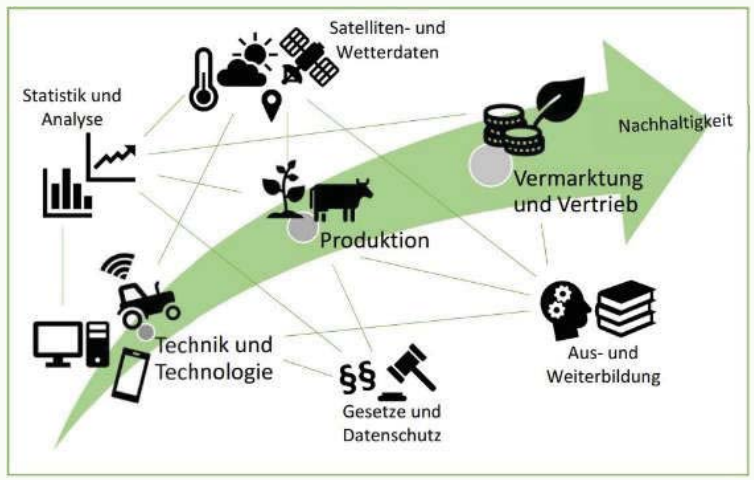

> Beschreibe das obige Prozessschaubild mit Fokus auf die Verbindungen.

$>$ Ergänze konkrete Praxisbeispiele zu den einzelnen Bereichen.

\section{A 5 Wenn die Entscheidung für einen Bio-Betrieb fällt ...}

Jeder land- und forstwirtschaftliche Betrieb fügt sich vielen gesetzlichen Auflagen. Biobetriebe haben speziellere Normen und Auflagen zu erfüllen, was die Produktion betrifft. Manche Verbände und Gütesiegel sind diesbezüglich sogar noch strenger.

> Erstelle eine kurze Präsentation, wie man Bio-Landwirt/in wird. Arbeite heraus, warum sich ein Umstieg lohnen könnte, wo Herausforderungen liegen und was die Digitalisierung dazu beitragen kann.

Die Landwirtschaft - Mai 2015, ab S. 43 https://bit.ly/2Lz7p5k

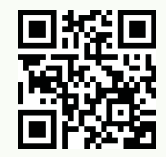

\section{A 6 Die Zukunft der Landwirtschaft}

Rollte noch gegen Ende des 19. Jahrhunderts der erste mit Benzin betriebene Traktor übers Feld, so steht die Landwirtschaft auch heute vor kleineren und größeren Umbrüchen. Wie kann es weitergehen?

Die Zukunft der Landwirtschaft 


\section{Bildnachweise}

M 1, M 3, A 6 Martin Anzengruber || M 2 Claas || A 1, A 4 Andrea Kircher (eigene Darstellung, 2018) || A 1, A 2, A 4 erstellt via learningsapps.org

\section{Literatur}

Berning, Frank (2015): Landwirtschaft 4.0: Chancen und Risiken. In: Top Agrar 11/2015, URL: https://www.topagrar.at/technik/Home-top-News-Landwirtschaft-4-0-Chancen-und-Risiken-2551932.html, Zugriff am 11.03 .2018

BMNT (Bundesministerium für Nachhaltigkeit und Tourismus) (2018a): Digitalisierung in der Landwirtschaft. Entwicklung, Heraus-forderungen und Nutzen der neven Technologien für die Landwirtschaft. URL: https://www.bmnt.gv.at/dam/jcr:f80a70323cb3-422b-8fa3-e3199d2caf4d/Digitalisierung\%20in\%20der\%20Landwirtschafti.pdf (Zugriff am 14.12.2018)

Bongardt, Katja (2016): Vernetzt vom Feld bis zum Verbraucher, In: Agrarzeitung 20 (2016), S. 10

Deter, A. (2015): Farming 4.0: Bleibt der Landwirt auf der Strecke? In: Top Agrar Online, 05.1 1.2015, URL: https://www.topagrar.com/news/Technik-Techniknews-Farming-4-0Bleibt-der-Landwirt-auf-der-Strecke-2598410.html, Zugriff am 11.03.2018

Deutsche Friedrich Wilhelm-Raiffeisen Gesellschaft (2018): Landwirtschaft 4.0, URL: https://raiffeisen2018.de/starke-idee/agrar/landwirtschaft-4-0, Zugriff am 11.03.2018

Deutsche Landwirtschafts-Gesellschaft (DLG) (2018): Digitale Landwirtschaft. Ein Positionspapier der DLG, URL: https://www.dlg.org/de/landwirtschaft/themen/technik/digitalisierung-arbeitswirtschaft-und-prozesstechnik/digitale-landwirtschaft/, Zugriff am 29.03.2018

Fritz, A. (2016): Digitale Landwirtschaft: Diese Vorteile sehen Landwirte, In: Agrarheute 2016, URL: https://www.agrarheute.com/management/digitale-landwirtschaftdiese-vorteile-sehen-landwirte-528356, Zugriff am 11.03.2018

Fuchs, S. H. (2017): Digitalisierung ist kein Selbstzweck. In: Bayerisches Landwirtschaftliches Wochenblatt, URL: https://www.agrarheute.com/wochenblatt/regionen/franken/digitalisierung-kein-selbstzweck-541205, Zugriff am 11.03 .2018

Göggerle, Th. (2017): Praktiker-Tagebuch zu Farming 4.0: Digital ackern. In: Agrarheute 2017, URL: https://www.agrarheute.com/technik/traktoren/praktiker-tagebuch-farming-40-digital-ackern-534060, Zugriff am 11.03 .2018

Grogan, A. (2012): Smart Farming, In: Engineering \& Technology, Juli 2012, S. 38-40

Landwirtschaftskammer NÖ (2015): Bio-ackerbau - richtig einsteigen. In: Die Landwirtschaft, Mai 2015, S. 43-56, URL: https://issuU.com/landwirtschaftskammer_noe/docs/die_landwirtschaft_mai_2015,Zugriff am 29.03.2018

Landwirtschaftskammer Österreich (2016): Agrarischer Ausblick Österreich 2025, URL: https://www.lko.at/media.php? filename=download=/2016.1 1.30/148049533266992.pdf\&rn=Agrarischer\%20Ausblick\%20Österreich\%202025.pdf, Zugriff am 31.08.2018

Leitner, A. (2008): AgrarGIS hilft Bäuerinnen und Bavern bei Flächenbewirtschaftung. In: Landwirt. Die Fachzeitschrift für die bäuerliche Familie, URL: https://www.landwirt.com/AgrarGIS-hilft-Baeverinnen-und-Bavern-bei-Flaechenbewirtschaftung,,5317,,Bericht.html, Zugriff am 11.03.2018

Lenge, R. (2017): Smart Farming. Wie die Landwirtschaft von der Digitalisierung profitiert, In: IM+io Fachmagazin, Ausgabe 2/2017, S. 54-57

Nadlinger, M. (2015): Parallelfahrsysteme, FJ Wieselburg „Innovative Agrartechnik“, URL: http://www.josephinum.at/fileadmin/content/BLT/6_News/2015-02-26_InnovativeAgrartechnik/01_NadlingerManfred.pdf, Zugriff am 29.03.2018

Weitbrecht, L. (2017): Im Visier: Feldarbeit 4.0, URL: https://vision.zf.com/site/magazine/de/articles_2816.html, Zugriff am 11.03 .2018

Weltzien, C. (2016): Digitale Landwirtschaft - oder warum Landwirtschaft 4.0 auch nur kleine Brötchen backt. In: Landtechnik 71 (2), 2016, S. 66-68

Prankl, H. (2016): Landwirtschaft 4.0: Neue Stufe der Vernetzung und Digitalisierung. In: Bavernzeitung 2016, URL: http://www.bavernzeitung.at/landwirtschaft-4-0-nevestufe-der-vernetzung-und-digitalisierung/, Zugriff am 11.03.2018

Spath, D. (Hrsg.), Ganschar, O., Gerlach, S., Hämmerle, M., Krause, T., Schlund, S. (2013): Produktionsarbeit der Zukunft - Industrie 4.0 (Studie). Stuttgart, Fraunhofer-Institut für Arbeitswirtschaft und Organisation, $150 \mathrm{~S}$. 